\title{
Monitoring olive oils quality and oxidative resistance during storage using an electronic tongue
}

\author{
Nuno Rodrigues a, b, Luís G. Dias ${ }^{\text {a, c }}$, Ana C.A. Veloso d, e , José Alberto Pereira a \\ António M. Peres ${ }^{\mathrm{f}, *}$ \\ a School of Agriculture, Polytechnic Institute of Bragança, Campus Santa Apolónia, 5300-253 Bragança, Portugal \\ ${ }^{\mathrm{b}}$ Universidad de Léon, Departamento de Ingeniería Agrária, Av. Portugal, $n^{\circ}$ 41, 24071 Léon, España, Spain \\ c CQ-VR, Centro de Química - Vila Real, University of Trás-os-Montes e Alto Douro, Apartado 1013, 5001-801 Vila Real, Portugal \\ d Instituto Politécnico de Coimbra, ISEC, DEQB, Rua Pedro Nunes, Quinta da Nora, 3030-199 Coimbra, Portugal \\ e CEB - Centre of Biological Engineering, University of Minho, Campus de Gualtar, 4710-057 Braga, Portugal \\ ${ }_{\mathrm{f}}^{\mathrm{f}}$ Laboratory of Separation and Reaction Engineering - Laboratory of Catalysis and Materials (LSRE-LCM), Escola Superior Agrária, Instituto Politécnico de \\ Bragança, Campus Santa Apolónia, 5300-253 Bragança, Portugal
}

\section{A R T I C L E I N F O}

\section{Article history:}

Received 13 April 2016

Received in revised form

6 June 2016

Accepted 3 July 2016

Available online 4 July 2016

\section{Keywords:}

Olive oils

Physicochemical quality parameters

Electronic tongue

Multiple linear regression

Simulated annealing algorithm

\begin{abstract}
A B S T R A C T
Monitoring olive oils oxidative stability and quality parameters (free acidity, peroxide values, $K_{232}$ and $K_{270}$ extinction coefficients) is needed to guarantee that, during storage, their levels remain within the legal thresholds enabling their commercialization as high-value extra-virgin olive oils. Physicochemical levels are assessed using time-consuming routine analytical reference techniques. In this work, the feasibility of a novel approach that merges an electronic tongue and chemometric tools, for monitoring extra-virgin olive oils' quality along one year of storage at dark or exposed to light is discussed. The results confirmed that physicochemical parameters varied with the storage lighting conditions and more significantly with time. Also, multiple linear regression models, using sub-sets of 22-28 sensors selected with a meta-heuristic simulated annealing algorithm, allow evaluating the storage time-evolution of olive oils' peroxide values, extinction coefficients and oxidative stabilities with satisfactory accuracy $\left(R^{2} \geq 0.98\right.$ and $\geq 0.96$, for leave-one-out and repeated $K$-fold cross-validation procedures, respectively). The capability of monitoring, in a single electrochemical assay, legal required quality parameters of olive oils, decreases considerable the analysis time and cost, allowing checking the compliance of extra-virgin olive oil quality with labeling. So, the use of electronic tongues for extra-virgin olive oil shelf-life assessment could be envisaged.
\end{abstract}

๑) 2016 Elsevier Ltd. All rights reserved.

\section{Introduction}

Extra-virgin olive oils (EVOO) are quite appreciated by consumers due to their quality, sensory attributes and health benefits. So, as pointed out in the literature, there still is a commercial need to develop fast, portable and low-cost analytical methods for guaranteeing olive oil commercial category namely to distinguishing EVOO from virgin and lampante olive oils (VOO and LOO, respectively). Olive oils physicochemical parameters have been shown as important markers for quality assessment and olive oil grade discrimination, minimizing the risk of incorrect or abusive

\footnotetext{
* Corresponding author.

E-mail address: peres@ipb.pt (A.M. Peres).
}

olive oils labeling (Garcia, Martins, \& Cabrita, 2013; Sinelli, Cerretani, Di Egidio, Bendini, \& Casiraghi, 2010). However, besides olive cultivar, edapho-climatic conditions, harvesting and technological procedures, EVOO's physicochemical quality is also greatly influenced by storage conditions, namely time, temperature, type of packing material, exposition to air and/or to light (Abbadi et al., 2014; Ayyad et al., 2015; Ben-Hassine et al., 2013; Bubola, Koprivnjak, Sladonja, \& Belobrajić, 2014; Caponio et al., 2013; Cossignani, Luneia, \& Damiani, 2007; Fadda et al., 2012; GómezAlonso, Mancebo-Campos, Salvador, \& Fregapane, 2007; Jabeur, Zribi, Abdelhedi, \& Bouaziz, 2015; Pristouri, Badeka, \& Kontominas, 2010). Indeed, the levels of olive oils physicochemical quality parameters, such as the ultra-violet light absorption extinction coefficients $\left(K_{232}\right.$ and $\left.K_{270}\right)$, free acidity (FA) and peroxide value (PV) may significantly increase during storage 
(Abbadi et al., 2014; Afaneh, Abbadi, Ayyad, Sultan, \& Kanan, 2013; Fadda et al., 2012; Jabeur et al., 2015; Mendéz \& Falqué, 2007; Rababah, Feng, Yang, Eriefej, \& Al-Omoush, 2011; Stefanoudaki, Willians, \& Harwood, 2010), those of the oxidative stability (OS) decrease (Stefanoudaki et al., 2010) and so, an olive oil classified as extra-virgin when bottled may suffer degradation during storage resulting in an inferior quality grade when purchase and consumed. In fact, olive oils quality changes are inevitable and start immediately after the olive oil extraction due to lipid oxidation, which may lead to rancidity (Ben-Hassine et al., 2013; Vacca, Del Caro, Poiana, \& Piga, 2006) or to hydrolytic degradations causing partial loss of healthy minor constituents (Dabbou et al., 2011). Therefore, new analytical methods aiming to ensure the compliance of olive oil quality with labeling is of utmost relevance for olive oils producers and consumers (Abbadi et al., 2014). This aim could be accomplished by the development of simple, green, user-friendly and low-cost analytical devices that could provide fast assessment and monitoring of the physicochemical quality parameters of olive oils, which could be implemented as complementary or alternative methods to the time-consuming classical analytical reference techniques. For example, this need as motivate the development of simple, expeditious and economic techniques compared to the expensive and time consuming classical chromatographic techniques, like the use of Fourier transform infrared (FTIR) spectroscopy combined with chemometrics as a rapid tool to predict phenol content and antioxidant activity of olive fruits and oils (Machado et al., 2015) or the use of Ion Mobility Spectrometry (IMS) for assessing the stability and quality of single-variety EVOO over storage (Garrido-Delgado et al., 2015). Several sensor approaches, based on the use of electronic tongues, noses and/or eyes (Etongues, E-noses and E-eyes, respectively), have been reported for olive oils qualitative and/or quantitative electrochemical characterization. These studies have proven the potential of single or fusion methodologies between E-tongues, E-noses and/or E-eyes regarding organoleptic characterization (Apetrei, Gutierez, Rodríguez-Méndez, \& de Saja, 2007; Apetrei, Apetrei, Villanueva, de Saja, \& Gutierrez-Rosales, 2010; Apetrei, GhasemiVarnamkhasti \& Apetrei, 2016; Apetrei, Rodríguez-Méndez, Parra, Gutierrez, \& de Saja, 2004; Rodríguez-Méndez, Apetrei, \& de Saja, 2010; Veloso, Dias, Rodrigues, Pereira, \& Peres, 2016), olive oil quality levels discrimination (Apetrei \& Apetrei, 2013; Apetrei, Rodríguez-Méndez, \& de Saja, 2005; Escuderos, Sánchez, \& Jiménez, 2011, 2010; García-González \& Aparicio, 2004; Oliveri, Baldo, Daniele, \& Forina, 2009), olive oil geographical origin (Apetrei et al., 2010; Cosio, Ballabio, Benedetti, \& Gigliotti, 2006; Haddi et al., 2013, 2011; Oliveri et al., 2009) or monovarietal olive oil classification according to olive cultivar (Cimato et al., 2006; Dias, Rodrigues, Veloso, Pereira, \& Peres, 2016a; Dias et al., 2014). Moreover, the feasibility of applying electrochemical devices for evaluating polyphenolic contents in olive oils have been successfully reported (Apetrei \& Apetrei, 2013; Rodríguez-Méndez, Apetrei, \& de Saja, 2008) as well for the capability of E-noses and/or E-tongues to indirectly qualitatively assess the oxidation of EVOO at different storage periods and conditions, allowing differentiating olive oil samples stored under different light conditions and storage time-periods (Cosio, Ballabio, Benedetti, \& Gigliotti, 2007). So, the implementation of cost-effective sensor-based devices for monitoring EVOO physicochemical quality along the commercialization line and storage, aiming to verify if their quality indexes still meet the required legal thresholds (FA $<0.8 \%$ oleic acid; $\mathrm{PV}<20 \mathrm{mEq} \mathrm{O} / \mathrm{kg} ; K_{232}<2.5$ and $K_{270}<0.22$ according to the Commission regulation (ECC) $n^{\circ} 2568 / 91$ ) for being commercialized as high-value EVOO, is still an on-going exciting and challenging research topic. In this work, the feasibility of applying an E-tongue (with nonspecific cross-sensitivity lipid membranes) and multiple linear regression (MLR) models, established using a simulated annealing (SA) variable selection algorithm, to simultaneously (i.e., in a single-run assay) quantify PV, $K_{232}$ and $K_{270}$ levels as well as oxidative stability (OS) of bottled EVOO, during one-year of storage $(0,3,6,9$ and 12 months) and under different lighting conditions (kept at dark or exposed to light) aiming to simulate usual commercial storage of olive oils, is discussed. So, the work aimed to demonstrate the possibility of applying the E-tongue as a fast and cost-effective novel strategy for quantifying quality parameters and OS values of olive oils in a single-run, expanding the demonstrated capability of the electrochemical device for qualitative and semiquantitative olive oils assessment, namely for monovarietal EVOO discrimination according to olive cultivar (Dias et al., 2016a, 2014; Peres, Veloso, Pereira, \& Dias, 2014) or the classification according to sensory intensity perception of positive organoleptic sensations (Veloso et al., 2016).

\section{Materials and methods}

\subsection{Olive oil samples}

Thirty six dark amber glass bottles of blend EVOOs, from the same lot, produced at the north of Portugal (Mirandela region), were studied. The selected lot was an extra virgin olive oil with the Protected Designation of Origin (PDO) qualification “Azeite de Trásos-Montes" PDO. These olive oils were obtained from olives (mainly from cultivars Cobrançosa and Verdeal Transmontana, with a 10\% percentage of olives of cv Madural, according to the producer information) collected at the initial maturation indexes (1-3) and extracted at low temperatures $\left(\sim 22{ }^{\circ} \mathrm{C}\right)$. Four olive oil's different bottles were analyzed immediately after packing, regarding to physicochemical parameters (FA, PV, $K_{232}$ and $K_{270}$ extinction coefficients as well as $\Delta K$ values, and $O S$ ) as well as electrochemically. The other 32 bottles were stored in a laboratory at ambient temperature (during the one-year of storage the temperature varied in the range of $17{ }^{\circ} \mathrm{C}$ to $25^{\circ} \mathrm{C}$ ) in conditions that tried to mimic realstorage conditions of supermarkets, during a one-year storage period (3, 6, 9 and 12 months; being 8 bottles picked and analyzed at each time-period). Also, two different lighting conditions were studied: 16 bottles were stored at dark, protected from any exposition to daylight or artificial light from a lamp; and, the other 16 bottles were stored in lab open shelves exposed to natural daylight (that entered throw 3 windows but without any direct exposition to sun) and also to artificial lightness from 8 fluorescent lamps (lamps Phillips TL-D36W/840) that remained lit $14 \mathrm{~h}$ per day, trying to mimic the environmental typical conditions of storage supermarket facilities. Each lamp provided a luminous flux of $3250 \mathrm{~lm}$ (according to the manufacturer information), which illuminated a $6 \times 9 \mathrm{~m}^{2}$ laboratory, corresponding to approximately 482 lux. At each storage period olive oils samples were also evaluated physicochemically and electrochemically. Throughout this work, lighting conditions will be coded as "Dark" and "Light" corresponding to olive oils stored in darkness and olive oil kept in shelf exposed to natural and artificial usual light. Concerning the storage date code T0 is used for fresh olive oil (not stored, analyzed just after being packed) and T3, T6, T9 and T12 for olive oils stored during 3, 6, 9 and 12 months.

\subsection{Olive oils physicochemical quality parameters and oxidative stability evaluation}

The olive oil's quality parameters assessed were the free acidity (FA), peroxide value (PV) and the specific coefficients of extinction at 232 and $270 \mathrm{~nm}\left(K_{232}, K_{270}\right.$, and $\left.\Delta K\right)$. All the mentioned quality parameters were determined according to European Union 
standard methods (Commission regulation (ECC) nº 2568/91). FA values are expressed in terms of oleic acid, since this acid is the major fatty acid found in olive oils (corresponding to 55\%-83\% of the total fatty acid content). The oxidative stability (OS) of each olive oil was also determined in a Rancimat 743 apparatus (Metrohm $\mathrm{CH}$, Switzerland). For these assays, to $3.00 \mathrm{~g}$ of olive oil heated at $120.0 \pm 1.6^{\circ} \mathrm{C}$ a flow rate of $20 \mathrm{~L} / \mathrm{h}$ of air (filtered, cleaned, and dried) was supplied. The resulting volatile compounds were collected in water, and the increasing water conductivity $(\mu \mathrm{S} / \mathrm{cm})$ was continuously measured. The time (in hours) taken to reach the conductivity inflection curve point was recorded, and corresponded to the OS value. All physicochemical assays were carried out in triplicate (i.e., 3 samples were collected from each olive oil bottle and analyzed).

\subsection{E-tongue device}

The E-tongue included the same two print-screen potentiometric arrays described by Dias et al. (2014). The electrochemical device contained 20 sensors (diameter: $3.6 \mathrm{~mm}$; thickness: $0.3 \mathrm{~mm}$ ) obtained from the combination of 4 different lipid additives (octadecylamine, oleyl alcohol, methyltrioctylammonium chloride and oleic acid; $\approx 3 \%$ ); 5 different plasticizers (bis(1butylpentyl) adipate, dibutyl sebacate, 2-nitrophenyl-octylether, tris(2-ethylhexyl)phosphate and dioctyl phenylphosphonate; $\approx 65 \%$ ) and high molecular weight polyvinyl chloride (PVC; $\approx 32 \%$ ). All reagents were from Fluka (minimum purity $\geq 97 \%$ ). The type of sensors and polymeric membrane compositions (relative percentage of additive, plasticizer and PVC) were selected based on a previous work (Dias et al., 2009) considering the satisfactory signal stability over time $(\% \mathrm{RSD}<5 \%)$ and repeatability $(0.5 \%<\%$ RSD $<15 \%$ ) towards the basic standard taste compounds (sweet, acid, bitter, salty and umami). Lipid polymeric membranes were used since they promote interactions with taste substances via electrostatic or hydrophobic interactions (Kobayashi et al., 2010). Each sensor was identified with a letter $\mathrm{S}$ (for sensor) followed by a code for the sensor array (1: or 2:) and the number of the membrane (1-20, corresponding to different combinations of plasticizer and additive used) as previously reported (Dias et al., 2014).

\subsection{E-tongue analysis: sample preparation and potentiometric assays}

Olive oils were extracted using water-ethanol solutions (80:20 $\mathrm{v} / \mathrm{v}$ ), to overcome the difficulty of carrying out electrochemical assays in viscous non-conductive liquids (Apetrei et al., 2010) and electrochemically analyzed as previously described (Dias et al., 2014). Ethanol was of analytical grade (Panreac, Barcelona) and deionized type II water was used in all electrochemical assays. For the electrochemical assays, samples were withdrawn from each olive oil bottle, which was previously smoothly shaken, and extracted with a solution of deionized water and ethanol (p.a.). In each assay, $10.00 \mathrm{~g}$ of olive oil were mixed to $100 \mathrm{~mL}$ of hydroethanolic solution during 5-10 min under strong agitation. This process allowed the extraction of polar compounds which are related to sensory sensations of olive oils (Veloso et al., 2016). The mixture was left at ambient temperature during $60 \mathrm{~min}$, after which, $40.0 \mathrm{~mL}(\times 2)$ of the supernatant solution was carefully removed and immediately analyzed with the E-tongue, during 5 min enabling to carried out several electrochemical scans, being usually retained the last one, which would correspond a pseudoequilibrium overall signal. To minimize the risk of overoptimistic prediction performance of multivariate models, the data split procedure used to set the training and validation sets, was carried using only one electrochemical "average" signal profile per olive oil (assumed as the olive oil specific fingerprint) in order to avoid that results from assays and replicas of the same olive oil could belong to both training and validation sets. Since assays were carried out during one year $(0,3,6,9$ and 12 months) to control the potentiometric signal drifts of the E-tongue sensors, a calibration standard hydroethanolic $\left(\mathrm{H}_{2} \mathrm{O}: \mathrm{EtOH}\right.$ : 80:20 v/v) solution containing gallic acid monohydrate $\left(\sim 1 \times 10^{-3} \mathrm{~mol} / \mathrm{L}\right.$, purchased from Sigma-Aldrich with a minimum purity $\geq 99 \%$ ) was analyzed before and after each olive oil measurement series. Signal drifts were solved by subtracting the signal profile recorded by the E-tongue device during the analysis of each olive oil sample by the average signal profile recorded for the gallic acid standard solution.

\subsection{Statistical analysis}

The possible interaction effect between the two main effects (i.e, lighting conditions $\times$ storage date) was assessed and interpreted using graphs of estimated marginal means for each parameter studied, instead of applying a two-way ANOVA, since the experimental design was unbalanced (i.e., for T0, the first level of storage date effect corresponding to just bottled olive oils, no sub-level of the lighting conditions effect could be considered). Based on the plots the significance of the interaction effect as well as the type of interaction (additive or non-additive/disordinal effect) was qualitatively evaluated: plots with parallel or non-parallel lines would indicate a non-significant or a significant interaction effect, respectively. In the latter case, non-crossing lines or crossing lines would point to additive or non-additive (disordinal) interaction effect. In the first situation (additive effect) main effects may be discussed separately. In the second case (non-additive effect) main effects cannot be individually interpreted. Generally speaking, main effects should not be discussed in the presence of a significant disordinal non-additive interaction (Field, 2009; Winer, Brown, \& Michels, 1991). When possible, the effects of storage conditions (lighting conditions or storage date) on EVOO's physicochemical parameters and sensorial sensations were evaluated separately by means of a t-Student test (for comparing lighting versus darkness stored conditions for each storage date) and by means of an oneway ANOVA followed, when appropriate, by the Tukey's post-hoc multi-comparison test, for assessing the effect of the storage date for olive oils kept in dark or exposed to light. Linear Pearson correlation coefficient ( $R$-Pearson) was applied to evaluate the existence of bivariate correlations within the olive oils' physicochemical parameters. The potential of the E-tongue device to quantitatively estimate the physicochemical quality parameters of olive oils, periodically analyzed during one-year of storage and under different lighting conditions, was evaluated using multiple linear regression (MLR), principal components regression (PCR) and partial least-squares (PLS) models. Detailed information regarding multivariate statistical tools can be found in the literature (Izenman, 2008; Miller \& Miller, 2010). For the MLR models, the best subsets of $K$ independent predictors among the 40 E-tongue potentiometric signals recorded were chosen using a metaheuristic simulated annealing (SA) variable selection algorithm (Bertsimas \& Tsitsiklis, 1993; Cadima, Cerdeira, \& Minhoto, 2004; Kirkpatrick, Gelatt, \& Vecchi, 1983) allowing to find optimal MLRSA models. The SA algorithm searches, iteratively, for a global minimum that optimizes a system with $k(\subseteq K)$ variables. The solutions of the current and the new subsets of $k$ variables are compared, using the tau2 quality criterion, which is a measure of the goodness of fitting. A new solution is randomly selected in the neighborhood of the current solution, being chosen if a better result is obtained. Usually, 10,000 attempts are used to select the best subset of variables (best model), starting the process of selecting the best subsets of variables on each trial, thus ensuring a greater 
confidence in finding a true optimal solution. In the present study, for each sub-set of sensors under evaluation (possible combinations of 2-39 sensors), the set of sensors chosen was the one that maximized tau2 value (Cadima et al., 2004). For PCR and PLS models the signals of the 40 E-tongue sensors were used to establish the optimal number of principal components (PCs) in each model, which was set equal to the number of PCs that would minimize the root mean square error (RMSE). The predictive performances of the MLR-SA, PCR and PLS models established were compared based on the leave-one-out cross-validation (LOO-CV) procedure. The approach that showed the better prediction capability was further evaluated using the repeated K-fold crossvalidation (K-fold-CV) procedure. This strategy was used aiming to minimize the risk of over-optimistic results usually reported with the LOO-CV (Dias et al., 2016a; Dias, Zelda, Veloso, \& Peres, 2016b). For the second cross-validation procedure, data was divided into $K$ subsets that allowed obtaining $K$ models, each one fitted considering $K-1$ subsets, as the training set, leaving out one of the subsets for the internal validation, to compute the predictive error for the obtained model (Venables \& Ripley, 2002). The number of K-folds was set equal to 7, enabling the formation of testing subsets with $15 \%$ of the initial data (i.e., at least 5 olive oil independent samples/bottles for the present work) thus allowing bias reduction. Also, by applying a repeated K-fold-CV procedure (with 10 repetitions), the uncertainty of the estimates could be significantly reduced. To normalize the weight of each variable in the final linear classification model, variable scaling and centering procedures were applied. The possibility of using the E-tongue method as tool for quantifying the classical physicochemical olive oil quality parameters was further checked by testing if the slope and intercept values for LOO-CV or repeated K-fold-CV procedures were equal to the theoretical expected values (one and zero, respectively), from a statistical point of view (Cadima et al., 2004), when representing the predicted values, estimated by the regression models versus the experimental data. All statistical analysis were performed using the Subselect (Cadima et al., 2004; Cerdeira, Silva, Cadima, \& Minhoto, 2012) and MASS (Venables \& Ripley, 2002) packages of the open source statistical program $R$ (version 2.15.1) at a significance level of $5 \%$.

\section{Results and discussion}

\subsection{Trend of EVOO's physicochemical quality parameters and positive sensorial attributes during storage}

Physicochemical quality parameters (FA, PV, $K_{232}, K_{270}, \Delta K$, and OS) of olive oils, stored at light or dark conditions, were evaluated quarterly, during 12 months, and further compared with the starting parameters of just bottled olive oils. As mentioned, since the experimental design was unbalanced, the existence of a statistical significant interaction effect (lighting conditions $\times$ storage date) was assessed and interpreted using graphs of means instead. Since $\Delta K$ values kept almost constant (varying from 0.00 to 0.01 ) regardless the storage date and lighting storage conditions, this parameter was not statistically analyzed. The results (data not shown) pointed out that: (i) for FA there was no significant interaction effect between the main effects (parallel lines in the graph of estimated marginal means); (ii) for PV, $K_{232}, K_{270}$ and OS the interaction effects were statistically significant (non-parallel lines) but, with the exception of the latter parameter, the interaction effects were additive (non-crossing lines) indicating that in those cases each main effect could be evaluated separately. Therefore, the influence of the storage date on EVOO's physicochemical parameters, regardless the lighting conditions, was further assessed using one-way ANOVA and the Tukey's test. The possible effect of the storage lighting conditions, at each storage date, was investigated using a $t$-Student test. The existence of similar time-evolution trends were discussed using $R$-Pearson linear correlation coefficients.

One-way ANOVA showed that, for each lighting storage condition, the storage date significantly affected, at a $5 \%$ significance level, FA, PV, $K_{232}, K_{270}$ and OS values ( $P$-values $\left.<0.0001\right)$. Also, except for $K_{270}$ coefficient, statistically similar linear time-evolution trends were found during storage time $(0.89 \leq R$-Pearson $\leq 0.999)$, regardless the lighting exposition. Based on Fig. 1 and on the results of the Tukey's test it can be stated that, in general, FA, $K_{232}, K_{270}$ levels of olive oils stored at light or dark significantly increase with the storage time, being more evident after 9 months of storage. The increase of FA, $K_{270}$ and PV over time is in accordance with previous studies (Afaneh et al., 2013; Ben-Hassine et al., 2013; Dabbou et al. 2011; Fadda et al., 2012; Rababah et al., 2011). $K_{270}$ increase may be attributed to the formation of secondary products due to autooxidation. PV increased during the first 3 months of storage and then remained almost constant, which can be due to the primary oxidation that occurs in the presence of air in the bottle headspace (and enhanced by the exposition to light) leading to the production of peroxides until an equilibrium is reached between the production and decomposition of peroxides to secondary products. On the other hand, the OS values of olive oils decrease during the storage period but only slightly, which may be attributed to their expected high total phenolic contents, promoted by the low maturation indexes (1-3) of the olives used in the production, the low extraction temperature $\left(\sim 22^{\circ} \mathrm{C}\right)$, and the olive cultivars used (cvs. Cobrançosa, Verdeal Transmontana and Madural ( 10\%)), which confer high resistance to oxidation (Sousa, Malheiro, Casal, Bento, \& Pereira, 2014, 2015). After one-year of storage, independently of the lighting conditions, the maximum mean FA and PV levels $(0.28 \pm 0.00 \%$ oleic acid and $7 \pm 2 \mathrm{mEq} \mathrm{O} / \mathrm{kg}$, respectively) of olive oils did not exceed the legal limits $(0.8 \%$ oleic acid and $20 \mathrm{mEq} \mathrm{O} / \mathrm{kg}$, respectively; ECC regulation $\mathrm{n}^{\circ} 2568 / 91$ ), which could be tentatively attributed to the expected initial high contents in phenolic compounds considering the specific olive cultivars used (Sousa et al., 2014, 2015). Contrary, and as reported in the literature (Afaneh et al., 2013; Ben-Hassine et al., 2013), after 9-12 months, olive oils stored at dark (mean $K_{232}$ values equal to $2.9 \pm 0.7$ ) or exposed to light (mean $K_{270}$ values $\geq 0.26 \pm 0.05$ ) exceeded the legal thresholds for EVOO classification (2.5 and 0.22, respectively; ECC regulation $\left.n^{\circ} 2568 / 91\right)$. The results of the $t$-Student analysis also pointed out that lighting storage conditions (i.e., dark or natural/ artificial light exposition) did not significantly affect olive oils' FA or OS along the one-year of storage. The similar OS found could be due to the use of dark amber glass bottles, which are known to minimize olive oils' quality degradation during storage and, also, because the light exposition was not intensive since it was achieved using fluorescent lamps ( 482 lux) that mimic normal supermarket storage environment. Contrary, PV, $K_{232}$ and $K_{270}$ levels of olive oils with the same storage time, were significantly affected by the lighting storage conditions. Unexpectedly, PV of olive oils stored at light were statistically greater than those of olive oils stored at dark although the absence of light should delayed peroxide decomposition (Afaneh et al., 2013; Fadda et al., 2012). Similarly, the exposition of olive oils to light significantly increased $K_{270}$ levels, oppositely to the findings of Ben-Hassine et al. (2013). Finally, as expected, olive oils stored under dark conditions showed significantly greater $K_{232}$ values than those exposed to light.

Moreover, statistically significant linear correlations $(P$ value $<0.05$ ) were found between some physicochemical quality parameters and the OS values, corresponding to changes that occurred during storage time under different lighting conditions. During storage (0-12 months), as previously reported (Rotondi 

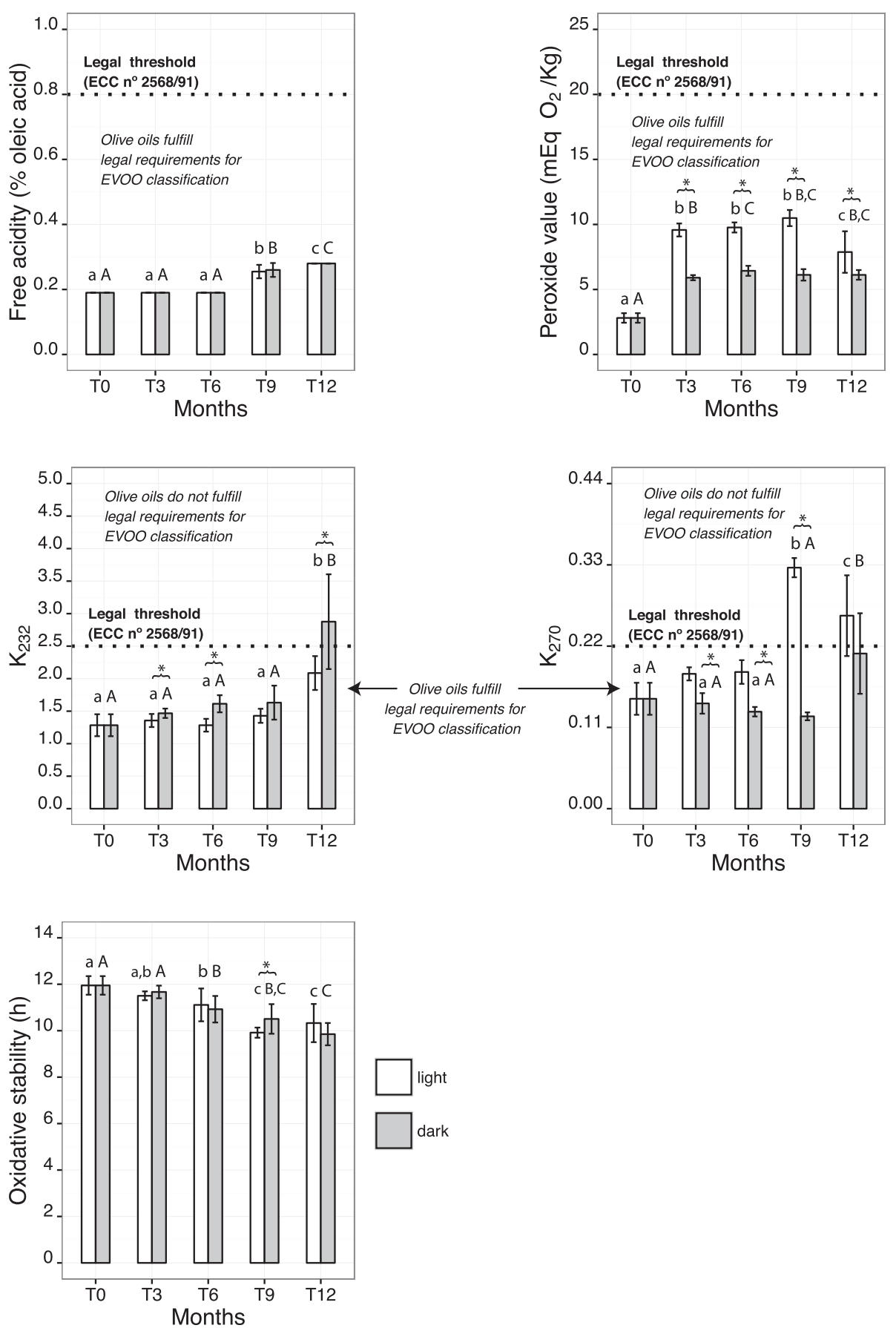

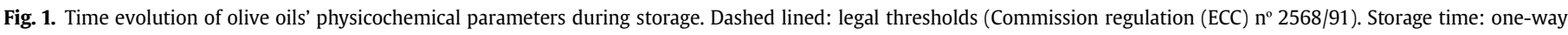

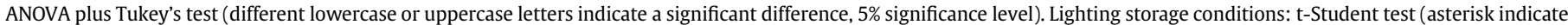
a significant statistical difference, $5 \%$ significance level).

et al., 2004), it was also found a significant negative correlation $(-0.97<R$-Pearson $<-0.86)$ of OS with FA (for light and dark conditions), with $K_{232}$ extinction coefficient (for dark condition) and for $K_{270}$ values (for light condition), i.e., olive oils with greater FA had lower OS, $K_{232}$ and $K_{270}$ levels.

Finally, it should be emphasized, as previously discussed, that based on the physicochemical quality parameters the initial highquality blend EVOOs do not comply "extra-virgin" label requirements after 9-12 months of storage, since at least two parameters $\left(K_{232}\right.$ and $\left.K_{270}\right)$ did not meet the required legal limits.
Indeed, the use of glass containers to store olive oil is not consensual in the literature, being described as suitable (GómezAlonso et al., 2007; Pristouri et al., 2010; Torres \& Maestri, 2006) and inappropriate (Garrido-Delgado et al., 2015; Mendéz \& Falqué, 2007; Samaniego-Sánchez, Oliveras-López, QuesadaGranados, Villalón-Mir, \& Serrana, 2012; Vekiari, Papadopoulou, \& Kiritsakis, 2007) packing material. This fact, show the pertinence of developing electrochemical devices for indirectly assessing shelf-life of stored EVOOs, by monitoring physicochemical quality parameters. 


\subsection{E-tongue signal profiles of olive oils during storage}

Each electrochemical analysis provided 40 potentiometric signals (for the 20 sensors and the respective replicas) varying from $-0.25 \mathrm{~V}$ to $+0.35 \mathrm{~V}$, showing each pair of sensor/sensor-replica slight signal differences due to the slight variations of the membrane composition, transparency and porosity attributed to the drop-by-drop technique applied, which may lead to the formation of inhomogeneous membranes (Dias et al., 2014). The magnitude of the signal profiles recorded with each sensor membrane of the Etongue could be tentatively attributed to the expected content in polar compounds of the hydroethanolic extracts (Veloso et al., 2016). Although the voltage signals were of similar magnitude for all sensors, to overcome possible undesired signal drift effects, considering the large analysis time interval (assays performed during a one-year period), the sensor signals recorded for each olive oil extract and time-period were corrected by subtracting the average signal $(+0.04 \mathrm{~V}$ to $+0.22 \mathrm{~V})$ recorded for a standard solution of gallic acid $\left(\sim 1 \times 10^{-3} \mathrm{~mol} / \mathrm{L}\right)$ at each time-period by each sensor. The final corrected sensor signals varied between $-0.34 \mathrm{~V}$ and $0.19 \mathrm{~V}$. Fig. 2 exemplifies the E-tongue signal average profiles (together with the related standard deviations) recorded with one of the two multisensory array (which comprised 20 different lipidic-based sensors). As can be inferred, olive oils with different storage time periods and stored at different lighting conditions showed different electrochemical profiles and signal magnitudes. The overall signal variability, which can be tentatively attributed to the changes of the physicochemical and/or sensorial attributes of olive oils that naturally occur during storage, may be further used to verify the capability of the E-tongue for monitoring the timeevolution of the olive oils' physicochemical quality parameters.

\subsection{Estimation of EVOO's physicochemical levels using the E-tongue device}

The potential of the E-tongue device to enable the quantification of quality physicochemical parameter levels during olive oil storage (namely PV, $K_{232}, K_{270}$ and OS) was evaluated (FA level was not considered due to the narrow concentration interval found for the olive oils analyzed: from 0.19 to 0.28 ). Indeed, it was meant to perform a quantitative study in opposition to the usual qualitative approaches, such as the exploratory unsupervised (e.g., principal component analysis and cluster analysis) and supervised (e.g., linear discriminant analysis) multivariate techniques, which have already proven to be suitable for olive oil storage conditions evaluation using electrochemical techniques (Cosio et al., 2007). The feasibility of the aimed quantitative strategy would enable to envisage a possible application of E-tongue devices as a quality control on-line tool in the olive oil field.

For that purpose, MLR-SA models based on the most informative sub-sets of sensors selected using the SA algorithm (among the 40 lipid polymeric sensors comprised in the E-tongue device), PCR and PLS models using all the 40 E-tongue sensors and a number of PCs that minimizes the RMSE, were established and their performance assessed using LOO-CV procedure. The results (Table 1) showed that MLR-SA approach possessed the best predictive capability for quantifying the 4 physicochemical parameters evaluated. Indeed, as pointed out by the results of Table 1 , the use of full-sensor multivariate statistical methods (PCR and PLS models) resulted in less reliable prediction models and could not minimize the possible noise effect of using more sensors ( 40 against $22-26$ sensors). The predictive performances of the MLR-SA models were further assessed by means of the repeated K-fold-CV procedure. The results from the two internal cross-validation procedures implemented (Figs. 3-6) show that the MLR-SA models established using the
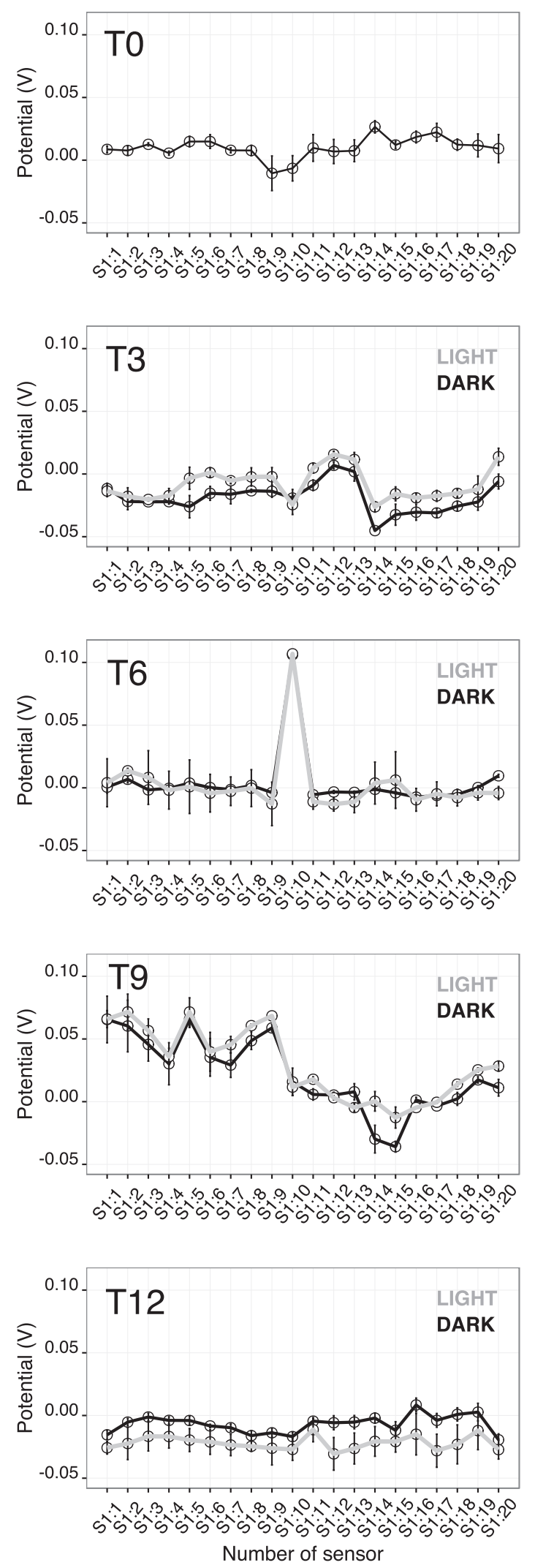

Fig. 2. E-tongue corrected mean signal profiles (error bars - related standard deviations) recorded during the potentiometric analysis of olive oils' hydroethanolic extracts along storage (storage time: T0, T3, T6, T9 and T12 - initial time and 3-12 months of storage; storage respectively; storage lighting conditions: dark - black full lines and light - grey full lines). 
Table 1

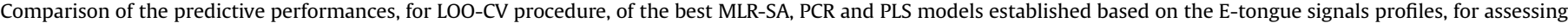

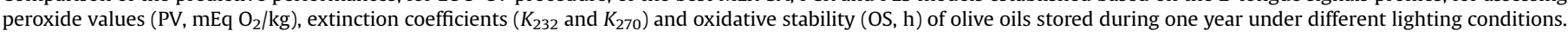

\begin{tabular}{|c|c|c|c|c|c|c|}
\hline \multirow[t]{3}{*}{ Physicochemical parameters } & \multicolumn{6}{|c|}{ Multivariate statistical models (results for LOO-CV procedure) } \\
\hline & \multicolumn{2}{|l|}{ MLR-SA } & \multicolumn{2}{|l|}{ PCR } & \multicolumn{2}{|l|}{ PLS } \\
\hline & $\mathrm{N}^{\circ}$ sensors & $R^{2}$ & $\mathrm{~N}^{\circ} \mathrm{PCs}$ & $R^{2}$ & $\mathrm{~N}^{\circ} \mathrm{PCS}$ & $R^{2}$ \\
\hline PV & 22 & 0.990 & 13 & 0.735 & 5 & 0.756 \\
\hline$K_{232}$ & 23 & 0.990 & 11 & 0.768 & 4 & 0.765 \\
\hline$K_{270}$ & 26 & 0.983 & 10 & 0.712 & 6 & 0.751 \\
\hline OS & 26 & 0.987 & 8 & 0.762 & 5 & 0.793 \\
\hline
\end{tabular}

PCs: principal components.
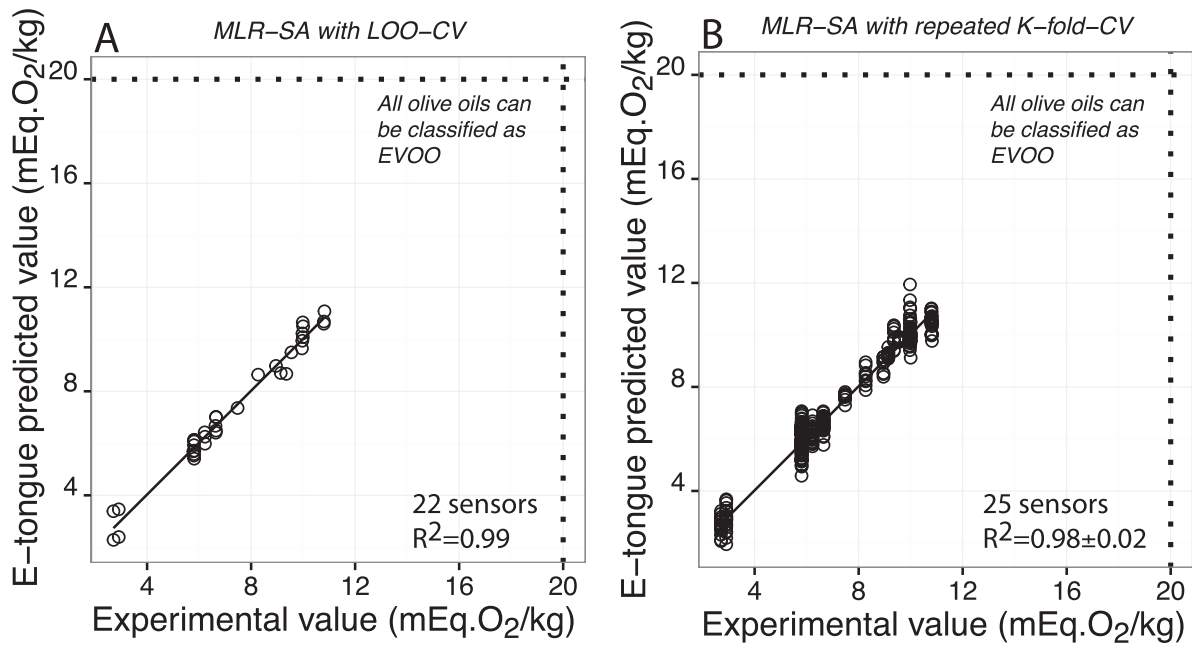

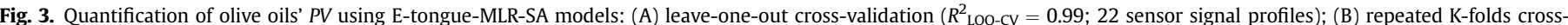

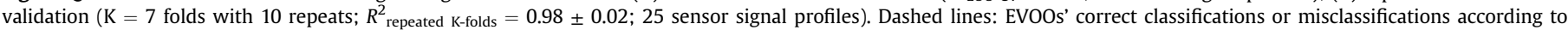
legal thresholds (Commission regulation (ECC) $\mathrm{n}^{\circ} 2568 / 91$ ).
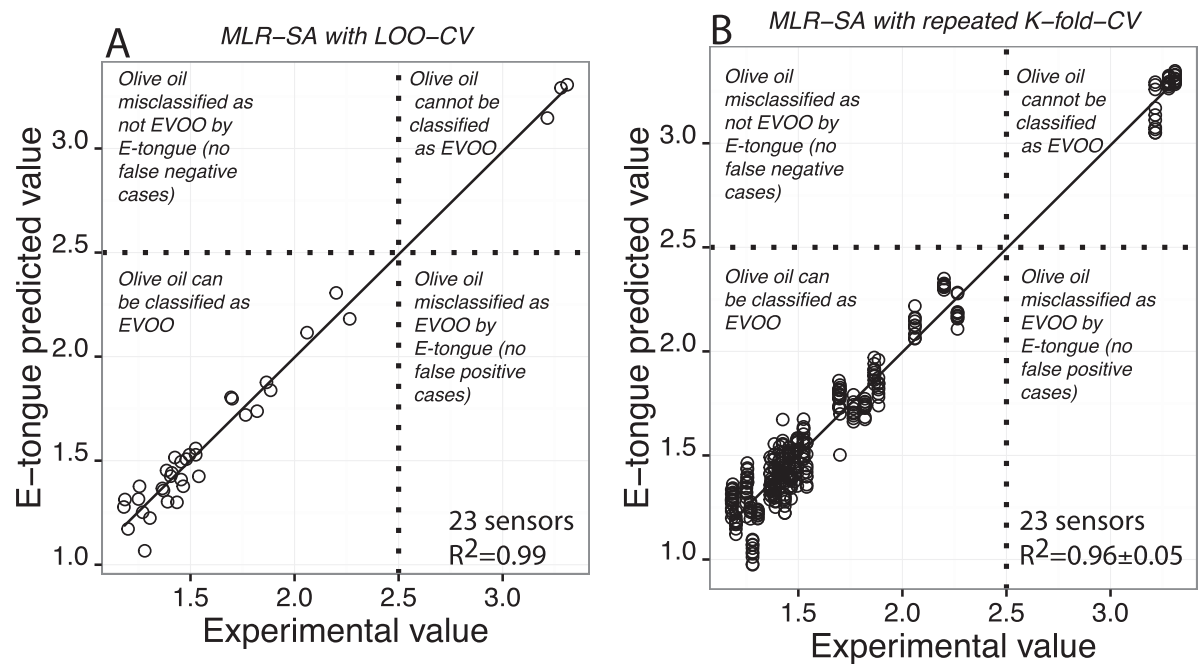

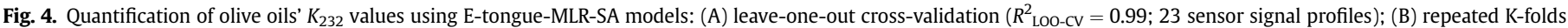

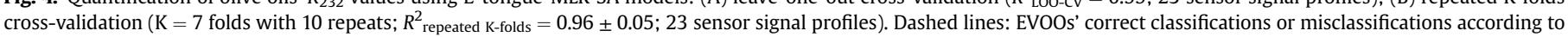
legal thresholds (Commission regulation (ECC) $n^{\circ} 2568 / 91$ ).

potentiometric signal profiles of 22-28 E-tongue sensors, depending on the parameter, enabled a satisfactory quantification of physicochemical quality parameters (LOO-CV: $R^{2}>0.98$; and, repeated K-fold-CV: $R^{2} \geq 0.96$ ), pointing out the possibility of using the E-tongue as a practical monitoring tool for evaluating the changes of key olive oil's quality physicochemical parameters during storage. As previously discussed, the repeated K-fold-CV procedure was used to minimize the risk of data over-fitting (which could result in over-optimistic model performance) and for that, the olive oils dataset was split into 7 folds (K-folds $=7$ ), meaning 

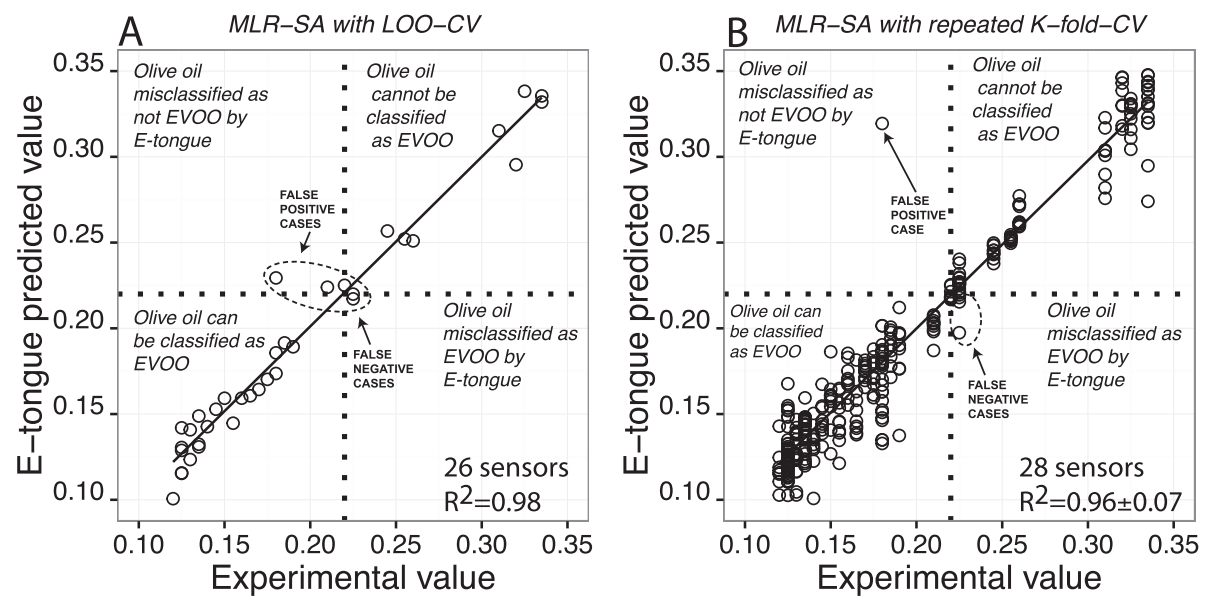

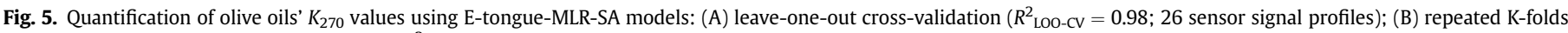

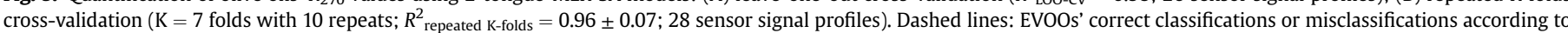
legal thresholds (Commission regulation (ECC) $n^{\circ} 2568 / 91$ ).
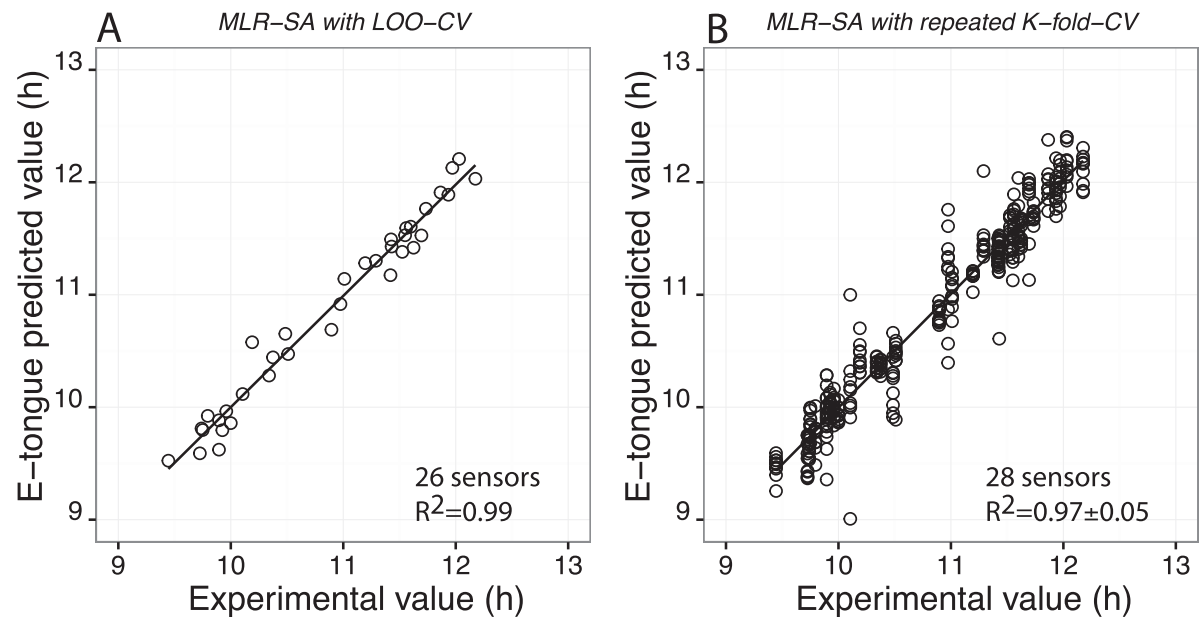

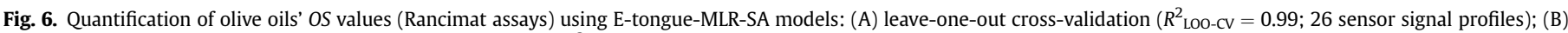
repeated K-folds cross-validation ( $\mathrm{K}=7$ folds with 10 repeats; $R_{\text {repeated K-folds }}^{2} 0.97 \pm 0.05$; 28 sensor signal profiles).

that at least $15 \%$ of independent data was used for internal validation during each one of the 10 repetition cycles, increasing the accuracy of the estimates and minimizing any bias effect. This quantification capability demonstrated by the E-tongue device could be tentatively attributed to the changes of nature and amount of the polar compounds extracted from the olive oils at each storage date, due to the natural occurrence of oxidation processes, affecting the intensity of olive oil sensorial characteristics (e.g., pungency, astringency and bitterness), which may be detected by the Etongue.

Also, the capability of quantifying three physicochemical quality parameters (PV, $K_{232}$ and $K_{270}$ ), which levels must be evaluated to verify the fulfillment of the legal requirements for classifying an olive oil as EVOO, represents a proof-of-principle that the electrochemical device may be applied as a complementary tool for olive oil analysis. Indeed, it can be easily verified (Fig. 3) that for all the olive oils analyzed during the one-year storage time, the estimated PV levels with the E-tongue were quite lower than the maximum legal value of $20 \mathrm{mEq} \mathrm{O} / 2 / \mathrm{kg}$, and so all olive oils would be classified as EVOO according to this physicochemical parameters likewise to the classification that would be made based on the classical tritation technique. Similarly, based on the $K_{232}$ values calculated from the E-tongue data (Fig. 4), olive oils would be correctly classified as EVOO or not ( $K_{232}$ values $\leq 2.5$ or $>2.5$, respectively) in total accordance with the conclusions that could be drawn based on the spectophotometric classical analysis. For both cases, the E-tongue/ MLR-SA quantification method did not reveal any false negative or false positive olive oil misclassification. On the contrary, the quantification of the $K_{270}$ levels using the E-tongue device (Fig. 5) led to some false negative olive oils classifications (i.e., EVOO misclassified: $\left(K_{270}\right)$ estimated E-tongue $\left.>0.22\right)$ and some false positive olive oils classifications (i.e., olive oils misclassified as EVOO: $\left.\left(K_{270}\right)_{\text {estimated E-tongue }}<0.22\right)$. However, the number of olive oils that could be misclassified based on the electrochemical analysis represented less than $15 \%$ of the 36 olive oil bottles analyzed during the one-year storage time ( 4 olive oils), which is quite satisfactory considering the reduction of the time and cost of the assay compared to the classical standard technique. Finally, the capability of estimating olive oils' OS from the E-tongue analysis (Fig. 6) may envisage a significant reduction of the analysis time reduction, since instead of carrying out a rancimat assay during a long period of time (hours or even days) an accurate estimate of the OS value could be electrochemically performed in few minutes.

The overall satisfactory performance was further demonstrated 
Table 2

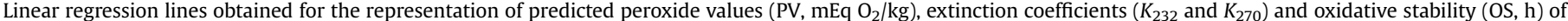

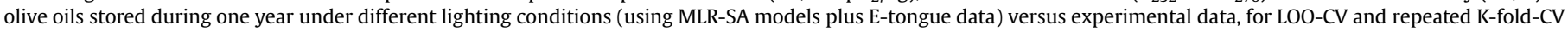
procedures: slope, intercept values and respective 95\% confidence intervals $(\mathrm{CI})$.

\begin{tabular}{|c|c|c|c|c|c|c|c|c|}
\hline \multirow{3}{*}{$\begin{array}{l}\text { Regression line } \\
\text { parameters }\end{array}$} & \multicolumn{8}{|c|}{ Physicochemical quality parameters of olive oils during storage (at dark and light conditions) } \\
\hline & \multicolumn{2}{|l|}{ PV } & \multicolumn{2}{|l|}{$K_{232}$} & \multicolumn{2}{|l|}{$K_{270}$} & \multicolumn{2}{|l|}{ OS } \\
\hline & LOO-CV & $\begin{array}{l}\text { Repeated K-fold- } \\
C V\end{array}$ & $\mathrm{LOO}-\mathrm{CV}$ & $\begin{array}{l}\text { Repeated K-fold- } \\
\text { CV }\end{array}$ & LOO-CV & $\begin{array}{l}\text { Repeated K-fold- } \\
\text { CV }\end{array}$ & LOO-CV & $\begin{array}{l}\text { Repeated K-fold- } \\
\text { CV }\end{array}$ \\
\hline$R^{2}$ & 0.990 & 0.970 & 0.990 & 0.959 & 0.983 & 0.903 & 0.987 & 0.944 \\
\hline Slope & 0.998 & 1.001 & 0.989 & 0.994 & 0.989 & 1.023 & 0.989 & 1.009 \\
\hline Slope $\mathrm{Cl}^{\mathrm{a}}$ & {$[0.949,1.047]$} & {$[0.982,1.019]$} & {$[0.939,1.039]$} & {$[0.976,1.012]$} & {$[0.924,1.053]$} & {$[0.988,1.058]$} & {$[0.933,1.046]$} & {$[0.983,1.034]$} \\
\hline Intercept & 0.039 & 0.022 & 0.018 & 0.009 & 0.003 & -0.005 & 0.106 & -0.093 \\
\hline Intercept $\mathrm{CI}^{\mathrm{b}}$ & $\begin{array}{l}{[-0.335,} \\
0.413]\end{array}$ & {$[-0.118,0.161]$} & $\begin{array}{l}{[-0.070} \\
0.106]\end{array}$ & {$[-0.022,0.040]$} & $\begin{array}{l}{[-0.010,} \\
0.016]\end{array}$ & {$[-0.012,0.002]$} & $\begin{array}{l}{[-0.511,} \\
0.722]\end{array}$ & {$[-0.372,0.185]$} \\
\hline
\end{tabular}

a $95 \%$ slope confidence interval.

b $95 \%$ intercept confidence interval.

for the two internal-validation procedures applied (LOO-CV and repeated K-fold-CV) since, as can be inferred from Figs. 3-6, plotting olive oil quality physicochemical levels estimated using the Etongue signal profiles and the previously established MLR-SA models versus the experimental data generated by the classical analytical techniques, linear straight lines were obtained (slope and intercept values and the 95\% confidence intervals are gathered in Table 2). These results demonstrate that, at $5 \%$ of significance level, the respective slopes and intercept values (of each regression line) are statistically equal to the theoretical expected values (slope equal to one; intercept equal to zero). These findings confirmed the robustness of the proposed MLR-SA models and their possible practical application. Indeed, the overall satisfactory quantitative performance achieved is indicative that the proposed approach could be implemented for routine olive oils quality analysis, allowing an accurate estimative of the most relevant physicochemical quality parameters as well as the OS in a 5 min single electrochemical run that requires a small amount of olive oil sample (10 g, 10 mL), which are major advantages compared to the time-consuming classical reference analytical techniques.

The quantitative potential of the E-tongue-MLR-SA method to simultaneous quantify PV, $K_{232}, K_{270}$ and OS values of olive oils, constitutes the main contribution of the present work and may be seen as a proof-of-concept of a novel applicability field of electrochemical-based strategies within olive oil quality control during storage. Nevertheless, the capability of simultaneously evaluating the FA levels of olive oils must be evaluated in future works in order to establish the E.-tongue device as a complementary/alternative tool for physicochemical quality assessment of olive oils.

\section{Conclusions}

The study carried out showed that, in general, the EVOOs' physicochemical quality parameters (FA, PV, $K_{232}$ and $K_{270}$ ) followed the expected time-evolution during storage, although the influence of lighting conditions on PV and $K_{232}$ values was contrary to the reported in the literature. On the other hand, it was also observed that some of the premium EVOOs studied, although stored in recommended dark amber bottles, suffered a degradation of the $K_{232}$ and $K_{270}$ quality parameters in such a level that no longer could be classified as EVOOs after 9-12 months of storage. This fact, which has also been described in the literature, points out the real need of quantitatively monitoring the levels of these legal required parameters throughout the olive oils' storage time in order to ensure the correctness of the olive oils label (and indirectly their shelf-life) as well as to enhance the consumers' confidence when purchasing this type of high-value food product.

In this context, the potentimetric E-tongue multi-sensor device coupled with MLR-SA models exhibited satisfactory predictive potential to assess $\mathrm{PV}, K_{232}, K_{270}$ and $\mathrm{OS}$ values of olive oils during one-year of storage. Globally, the olive oils' physicochemical levels assessed by the E-tongue procedure were in agreement with those determined using the time-consuming analytical standard techniques. Nevertheless, it should be remarked that $K_{270}$ levels evaluated from the E-tongue may lead to some, although few, EVOO misclassifications (both false negative and positive cases). Finally, since a single electrochemical assay enable the simultaneously quantification of physicochemical olive oil parameters, reducing the analysis time and cost, the application of this kind of electrochemical device in the olive oil industry may be foreseen in a near future as a practical and accurate quality control tool. However, prior to the possible implementation of this proof-of-concept, a significant larger number of olive oil samples must be evaluated.

\section{Acknowledgments}

This work was co-financed by Project POCI-01-0145-FEDER006984 - Associated Laboratory LSRE-LCM funded by FEDER funds through COMPETE2020 - Programa Operacional Competitividade e Internacionalização (POCI), by national funds through FCT Fundação para a Ciência e a Tecnologia; and by the strategic funding of UID/BIO/04469/2013 unit. Nuno Rodrigues thanks FCT, POPHQREN and FSE for the Ph.D. Grant (SFRH/BD/104038/2014).

\section{References}

Abbadi, J., Afaneh, I., Ayyad, Z., Al-Rimawi, F., Sultan, W., \& Kanaan, K. (2014). Evaluation of the effect of packaging materials and storage temperatures on quality degradation of extra virgin olive oil from olives grown in Palestine. American Journal of Food Science and Technology, 2, 162-174.

Afaneh, I. A., Abbadi, J., Ayyad, Z., Sultan, W., \& Kanan, K. (2013). Evaluation of selected quality degradation indices for palestinian extra virgin olive oil bottled in different packaging materials upon storage under different lighting conditions. Journal of Food Science and Engineering, 3, 267-283.

Apetrei, I. M., \& Apetrei, C. (2013). Voltammetric e-tongue for the quantification of total polyphenol content in olive oils. Food Research International, 54, 2075-2082.

Apetrei, C., Apetrei, I. M., Villanueva, S., de Saja, J. A., Gutierrez-Rosales, F., et al, (2010). Combination of an e-nose, an e-tongue and an e-eye for the characterisation of olive oils with different degree of bitterness. Analytica Chimica Acta, 663, 91-97.

Apetrei, C., Ghasemi-Varnamkhasti, M., \& Apetrei, I. M. (2016). Chapter 27-Olive oil and combined electronic nose and tongue. In M. R. Mendez (Ed.), Electronic noses and tongues in food science (pp. 277-289). , Oxford: Academic Press, Elsevier Inc.

Apetrei, C., Gutierez, F. Rodríguez-Méndez, M. L., \& de Saja, J. A. (2007). Novel method based on carbon paste electrodes for the evaluation of bitterness in extra virgin olive oils. Sensors and Actuators B, 121, 567-575.

Apetrei, C., Rodríguez-Méndez, M. L., \& de Saja, J. A. (2005). Modified carbon paste 
electrodes for discrimination of vegetable oils. Sensors and Actuators B, 111-112, 403-409.

Apetrei, C., Rodríguez-Méndez, M. L., Parra, V., Gutierrez, F., \& de Saja, J. A. (2004). Array of voltammetric sensors for the discrimination of bitter solutions. Sensors and Actuators B, 103, 145-152.

Ayyad, Z., Valli, E., Bendini, A., Adrover-Obrador, S., Femenia, A., \& Toschi, T. G. (2015). Extra-virgin olive oil stored in different conditions: Focus on diglycerides. Italian Journal of Food Science, 27, 166-172.

Ben-Hassine, K., Taamalli, A., Ferchichi, S., Mlaouah, A., Benincasa, C., Romano, E., et al. (2013). Physicochemical and sensory characteristics of virgin olive oils in relation to cultivar, extraction system and storage conditions. Food Research International, 54, 1915-1925.

Bertsimas, D., \& Tsitsiklis, J. (1993). Simulated annealing. Statistical Science, 8, 10-15.

Bubola, K. B., Koprivnjak, O., Sladonja, B., \& Belobrajić, I. (2014). Influence of storage temperature on quality parameters, phenols and volatile compounds of Croatian virgin olive oils. Grasas y Aceites, 65, e034.

Cadima, J., Cerdeira, J. O., \& Minhoto, M. (2004). Computational aspects of algorithms for variable selection in the context of principal components. Computational Statistics \& Data Analysis, 47, 225-236.

Caponio, F., Paradiso, V. M., Bilancia, M. T., Summo, C., Pasqualone, A., \& Gomes, T. (2013). Diacylglycerol isomers in extra virgin olive oil: Effect of different storage conditions. Food Chemistry, 140, 772-776.

Cerdeira, J. O., Silva, P. D., Cadima, J., \& Minhoto, M. (2012). Subselect: Selecting variable subsets. $\mathrm{R}$ package version $0.12-2$ http://CRAN.R-project.org/ package $=$ subselect.

Cimato, A., Dello-Monaco, D., Distante, C., Epifani, M., Siciliano, P., Taurino, A. M., et al. (2006). Analysis of single-cultivar extra virgin olive oils by means of Electronic Nose and HS-SPME/GC/MS methods. Sensors and Actuators B, 114, 674-680.

Commission regulation (ECC) $\mathrm{n}^{\circ} 2568 / 91$ : On the characteristics of olive oil and olive-pomace oil and on the relevant methods of analysis. Official Journal of the European Union, L248, (1991), 1-82, and all subsequent amendments.

Cosio, M. S., Ballabio, D., Benedetti, S., \& Gigliotti, C. (2006). Geographical origin and authentication of extra virgin olive oils by an electronic nose in combination with artificial neural networks. Analytica Chimica Acta, 567, 202-210.

Cosio, M. S., Ballabio, D., Benedetti, S., \& Gigliotti, C. (2007). Evaluation of different storage conditions of extra virgin olive oils with an innovative recognition tool built by means of electronic nose and electronic tongue. Food Chemistry, 101, 485-491.

Cossignani, L., Luneia, R. M., \& Damiani, P. (2007). Analysis of isomeric diacylglycerolic classes to evaluate the quality of olive oil in relation to storage conditions. European Food Research and Technology, 224, 379-383.

Dabbou, S., Gharbi, I., Dabbou, S., Brahmi, F., Nakbi, A., \& Hammami, M. (2011). Impact of packaging material and storage time on olive oil quality. African Journal of Biotechnology, 10, 16937-16947.

Dias, L. G., Fernandes, A., Veloso, A. C. A., Machado, A. A. S. C., Pereira, J. A., \& Peres, A. M. (2014). Single-cultivar extra virgin olive oil classification using a potentiometric electronic tongue. Food Chemistry, 160, 321-329.

Dias, L. G., Peres, A. M., Veloso, A. C. A., Reis, F. S., Vilas Boas, M., \& Machado, A. A. S. C. (2009). An electronic tongue taste evaluation: Identification goat milk adulterations with bovine milk. Sensors and Actuators B, 136, 209-217.

Dias, L. G., Rodrigues, N., Veloso, A. C. A., Pereira, J. A., \& Peres, A. M. (2016a). Monovarietal extra-virgin olive oil classification: A fusion of human sensory attributes and an electronic tongue. European Food Research Technology, 242, 259-270.

Dias, L. G., Zelda, A., Veloso, A. C. A., \& Peres, A. M. (2016b). Electronic tongue: A versatile tool for mineral and fruit-flavored waters recognition. Journal of Food Measurement and Characterization, 10, 264-273.

Escuderos, M. E., Sánchez, S., \& Jiménez, A. (2010). Virgin olive oil sensory evaluation by an artificial olfactory system, based on Quartz Crystal Microbalance (QCM) sensors. Sensors and Actuators B, 147, 159-164.

Escuderos, M. E., Sánchez, S., \& Jiménez, A. (2011). Quartz Crystal Microbalance (QCM) sensor arrays selection for olive oil sensory evaluation. Food Chemistry, $124,857-862$.

Fadda, C., Del Caro, A., Sanguinetti, A. M., Urgeghe, P. P., Vacca, V., Arca, P. P., et al. (2012). Changes during storage of quality parameters and in vitro antioxidant activity of extra virgin monovarietal oils obtained with two extraction technologies. Food Chemistry, 134, 1542-1548.

Field, A. (2009). Discovering statistics using IBM SPSS statistics (4 $4^{\text {th }}$ ed.). University of Sussex: SAGE Publications Ltd.

García-González, D. L., \& Aparicio, R. (2004). Classification of different quality virgin olive oils by metal-oxide sensors. European Food Research Technology, 218, 484-487.

Garcia, R., Martins, N., \& Cabrita, M. J. (2013). Putative markers of adulteration of extra virgin olive oil with refined olive oil: Prospects and limitations. Food Research International, 54, 2039-2044.

Garrido-Delgado, R., Dobao-Prieto, M. M., Arce, L., Aguilar, J., Cumplido, J. L., \& Valcárcel, M. (2015). Ion mobility Spectrometry versus classical physicochemical analysis for assessing the shelf life of extra virgin olive oil according to container type and storage conditions. Journal of Agricultural and Food Chemistry, 63, 2179-2188.

Gómez-Alonso, S., Mancebo-Campos, V., Salvador, M. D., \& Fregapane, G. (2007). Evolution of major and minor components and oxidation indices of virgin olive oil during 21 months storage at room temperature. Food Chemistry, 100, 36-42. Haddi, Z., Alami, H., El Bari, N., Tounsi, M., Barhoumi, H., Maaref, A., et al. (2013). Electronic nose and tongue combination for improved classification of Moroccan virgin olive oil profiles. Food Research International, 54, 1488-1498.

Haddi, Z., Amari, A., Ali, A. O., El Bari, N., Barhoumi, H., Maaref, A., et al. (2011) Discrimination and identification of geographical origin virgin olive oil by an enose based on MOS sensors and pattern recognition techniques. Procedia Engineering, 25, 1137-1140.

Izenman, A. J. (2008). Modern multivariate statistical Techniques: Regression, classification, and manifold learning (2nd ed., pp. 107-122). New York: Springer.

Jabeur, H., Zribi, A., Abdelhedi, R., \& Bouaziz, M. (2015). Effect of olive storage conditions on Chemlali olive oil quality and the effective role of fatty acids alkyl esters in checking olive oils authenticity. Food Chemistry, 169, 289-296.

Kirkpatrick, S., Gelatt, C. D., \& Vecchi, M. P. (1983). Optimization by simulated annealing. Science, 220, 671-680.

Kobayashi, Y., Habara, M., Ikezazki, H., Chen, R., Naito, Y., \& Toko, K. (2010). Advanced taste sensors based on artificial lipids with global selectivity to basic taste qualities and high correlation to sensory scores. Sensors, 10, 3411-3443.

Machado, M., Machado, N., Gouvinhas, I., Cunha, M., de Almeida, J. M. M. M., \& Barros, A. I. R. N. A. (2015). Quantification of chemical characteristics of olive fruit and oil of cv Cobrançosa in two ripening stages using MIR spectroscopy and chemometrics. Food Analytical Methods, 8, 1490-1498.

Mendéz, A. I., \& Falqué, A. (2007). Effect of storage time and container type on the quality of extra-virgin olive oil. Food Control, 18, 521-529.

Miller, J., \& Miller, J. C. (2010). Statistics and chemometrics for analytical chemistry (6th ed., pp. 231-235). Harlow: Prentice Hall.

Oliveri, P., Baldo, M. A., Daniele, S., \& Forina, M. (2009). Development of a voltammetric electronic tongue for discrimination of edible oils. Analytical and Bioanalytical Chemistry, 395, 1135-1143.

Peres, A. M., Veloso, A. C. A., Pereira, J. A., \& Dias, L. G. (2014). Electrochemical multisensors device coupled with heuristic or meta-heuristic selection algorithms for single-cultivar olive oil classification. Procedia Engineering, 87, 192-195.

Pristouri, G., Badeka, A., \& Kontominas, M. G. (2010). Effect of packaging material headspace, oxygen and light transmission, temperature and storage time on quality characteristics of extra virgin olive oil. Food Control, 21, 412-418.

Rababah, T. M., Feng, H., Yang, W., Eriefej, K., \& Al-Omoush, M. (2011). Effects of type of packaging material on physicochemical and sensory properties of olive oil. International Journal of Agricultural and Biological Engineering, 20, 66-72.

Rodríguez-Méndez, M. L., Apetrei, C., \& de Saja, J. A. (2008). Evaluation of the polyphenolic content of extra virgin olive oils using an array of voltammetric sensors. Electrochimica Acta, 53, 5867-5872.

Rodríguez-Méndez, M. L., Apetrei, C., \& de Saja, J. A. (2010). Chapter 57-Electronic tongues purposely designed for the organoleptic characterization of olive oils olives. In V. Preedy, \& R. R. Watson (Eds.), Olives and olive oil health and disease prevention (pp. 525-532). Oxford: Academic Press, Elsevier Inc.

Rotondi, A., Bendini, A., Cerretani, L., Mari, M., Lercker, G., \& Toschi, T. G. (2004). Effect of Olive Ripening Degree on the Oxidative Stability and Organoleptic Properties of Cv. Nostrana di Brisighella Extra Virgin Olive Oil. Journal of Agricultural and Food Chemistry, 52, 3649-3654.

Samaniego-Sánchez, C., Oliveras-López, M. J., Quesada-Granados, J. J., VillalónMir, M., \& Serrana, H. L.-G. (2012). Alterations in picual extra virgin olive oils under different storage conditions. European Journal of Lipid Science and Technology, 114, 194-204.

Sinelli, N., Cerretani, L., Di Egidio, V., Bendini, A., \& Casiraghi, E. (2010). Application of near (NIR) infrared and mid (MIR) infrared spectroscopy as a rapid tool to classify extra virgin olive oil on the basis of fruity attribute intensity. Food Research International, 43, 369-375.

Sousa, A., Malheiro, R., Casal, S., Bento, A., \& Pereira, J. A. (2014). Antioxidant activity and phenolic composition of $\mathrm{Cv}$. Cobrançosa olives affected through the maturation process. Journal of Functional Foods, 11, 20-29.

Sousa, A., Malheiro, R., Casal, S., Bento, A., \& Pereira, J. A. (2015). Optimal harvesting period for cvs. Madural and Verdeal Transmontana, based on antioxidant potential and phenolic composition of olives. LWT - Food Science and Technology, 62, 1120-1126.

Stefanoudaki, E., Willians, M., \& Harwood, J. (2010). Changes in virgin olive oil characteristics during different storage conditions. European Journal of Lipid Science and Technology, 112, 906-914.

Torres, M. M., \& Maestri, D. M. (2006). Chemical composition of Arbequina virgin olive oil in relation to extraction and storage conditions. Journal of the Science of Food and Agriculture, 86, 2311-2317.

Vacca, V., Del Caro, A., Poiana, M., \& Piga, A. (2006). Effect of storage period and exposure conditions on the quality of Bosana extra-virgin olive oil. Journal of Food Quality, 29, 139-150.

Vekiari, S. A., Papadopoulou, P., \& Kiritsakis, A. (2007). Effect of processing and commercial storage conditions on the extra virgin olive oil quality indexes. Grasas y Aceites, 58, 237-242.

Veloso, A. C. A., Dias, L. G., Rodrigues, N., Pereira, J. A., \& Peres, A. M. (2016). Sensory intensity assessment of olive oils using an electronic tongue. Talanta, 146 585-593.

Venables, W. N., \& Ripley, B. D. (2002). Modern applied statistics with S (statistics and computing) (4th ed.). New York: Springer.

Winer, B. J., Brown, D. R., \& Michels, K. M. (1991). Statistical principles in experimental design (3rd ed.). New York: McGraw-Hill. 